\title{
Contenido audiovisual y enseñanza universitaria a distancia: una propuesta metodológica
}

\author{
Ana Eva RodríGuez Bravo \\ Universidad Nacional de Educación a Distancia (UNED) \\ anaeva.rodriguez@edu.uned.es \\ Beatriz TASEnde MAÑá \\ Universidad Nacional de Educación a Distancia (UNED) \\ btasende@madrid.uned.es \\ Ángel Luis González Olivares \\ Universidad Nacional de Educación a Distancia (UNED) \\ algonzalez@edu.uned.es \\ Álvaro Muelas Plaza \\ Centro Universitario Villanueva \\ amuelas@villanueva.edu \\ Ángel de Juanas Oliva \\ Universidad Nacional de Educación a Distancia (UNED) \\ adejuanas@edu.uned.es
}

\begin{abstract}
Resumen:
El actual paradigma de las nuevas tecnologías posibilita acciones educativas orientadas al desarrollo nuevos espacios de comunicación y la distribución abierta de medios audiovisuales para la enseñanza. En este artículo, se presenta una propuesta metodológica para la incorporación del contenido audiovisual en la enseñanza universitaria a distancia. Se desarrolla durante el año 2013 en el marco de la asignatura "El aprendizaje de las personas adultas en la sociedad del conocimiento" que se imparte en el Máster de Intervención Educativa en Contextos Sociales de la Facultad de Educación (UNED).
\end{abstract}

Palabras clave: medios audiovisuales; enseñanza superior; enseñanza a distancia; metodología.

\section{Audiovisual content and distance teaching university: a methodological proposal}

\begin{abstract}
:
The current paradigm of new technologies makes educational activities aimed at developing new spaces for communication and open distribution of audiovisual media for teaching. In this paper, we present a methodology for the integration of audiovisual content to university distance. It takes place during the year 2013 under the subject "The adult learning in the knowledge society" that is taught in the Master of Educational Intervention on Social Contexts of the Faculty of Education (UNED).
\end{abstract}

Key Words: audiovisual aid; higher education; distance study; methodology. 


\section{Referencia normalizada:}

Rodríguez Bravo, A.E., Tasende Mañá, B., González Olivares, A.L., Muelas Plaza, A. y De Juanas Oliva, A. (2014): Contenido audiovisual y enseñanza universitaria a distancia: una propuesta metodológica. Historia y Comunicación Social. Vol. 19. Núm. Especial Enero. Págs. 235-251.

Sumario 1. Introducción. 2. Metodología. 3. Evaluación de la propuesta metodológica. 3.1. Datos cuantitativos. 3.2. Datos cualitativos. 4. Conclusiones. 5. Referencias bibliográficas.

\section{Introducción}

Las nuevas tecnologías han realizado cambios circunstanciales en nuestra vida porque la forma de vivir se ha reeducado tecnológicamente. La evolución ha sido tan rápida que, casi sin darnos cuenta, se ha pasado de meros receptores a sujetos interactivos con la información que nos rodea. El aprendizaje ha progresado junto con nuestra conducta, de tal modo se piensa en el aprender a aprender porque se requiere estar actualizados.

A finales de los noventa, Delors (1996) comentó que quedaba un claro camino por recorrer sustentado en la educación a lo largo de la vida y basado en cuatro pilares: aprender a conocer, aprender a hacer, aprender a vivir juntos y aprender a ser. Pero para poder aprender se debe conocer que aquello que se aprende debe ser adquirido por aptitud e idoneidad, y esa nueva meta consiste en adquirir competencias. Sevillano (2009, Pág. 6) señala que: "las competencias suponen valores, actitudes y motivaciones, además de conocimientos, habilidades y destrezas". En la Sociedad del Conocimiento hablar de competencias se puede equiparar a objetivos referentes que todas las personas precisan para su desarrollo personal y profesional. Entre ellas la competencia digital que exige una alfabetización digital, entendida como "un conjunto de competencias necesarias para el uso y dominio de las nuevas tecnologías" (Sevillano 2009, Pág. 11).

Para Lozano y Covelo (2009, Pág. 56), la Sociedad del Conocimiento tiene una "proyección inevitable en la educación". De tal manera, tiene el compromiso de reinventarse, porque es un proceso inevitable del progreso y desarrollo humano. Por tanto, la educación trata de deliberar sobre la revolución que ha originado la aparición de las nuevas tecnologías informáticas, que han llevado consigo una aceleración de la obtención y el uso de datos de información. Desde hace años, se vive en una cultura audiovisual que también lo es de la imagen, que requiere de una "educación audiovisual" en la que se complemente la pedagogía y la comunicación (Masterman, 1993).

Los recursos didácticos audiovisuales tienen una gran incidencia en educación. En el actual paradigma de las nuevas tecnologías, se han potenciado múltiples acciones educativas que amplían las posibilidades de crear nuevos entornos de comunicación y distribuir abiertamente medios audiovisuales para la enseñanza (Area y Ribeiro, 2012). Si bien, estos recursos suelen percibirse como añadidos a los escenarios educativos, con la función de originar un cierto virtuosismo estético o de recompensa 
educativa, sin tener en cuenta todo el potencial que pueden ofrecer (Cabero, 2004). Para lograr todo el potencial, se debe predisponer de escenarios de aprendizaje que contemplen estrategias y técnicas didácticas de primer orden. Para ello, se ha de dotar de significado al medio en función de los objetivos de aprendizaje pretendidos.

En este marco sociocultural a continuación se presenta una propuesta metodológica para la incorporación del contenido audiovisual en la enseñanza a distancia que se ha desarrollado en el marco de una asignatura de postgrado de un Máster ajustado a los planteamientos del Espacio Europeo de Educación Superior.

\section{Metodología}

La propuesta se ha desarrollado en torno al diseño, desarrollo y evaluación de la actividad Vínculos, con la cual se ha buscado tener en cuenta las creencias y actitudes de los estudiantes sobre los contenidos de aprendizaje de la materia, así como propiciar la aplicación de los contenidos a una práctica situada en el contexto neutro de un vídeo educativo. La hipótesis de trabajo ha sido que la actividad Vínculos favorecería la adquisición, procesamiento y control del aprendizaje de los estudiantes. De acuerdo con este planteamiento, la propuesta metodológica tuvo los siguientes objetivos: 1) potenciar el modelo de evaluación continua de la asignatura; 2) ampliar el espacio virtual de la asignatura ofreciendo contenidos audiovisuales; 3 ) elaborar un instrumento de evaluación que permitiese revisar la eficiencia de la actuación docente desde el punto de vista de los estudiantes; y 4) evaluar el proceso, así como realizar propuestas de mejora referidos a la tarea y al espacio virtual.

El diseño de la actividad se inició con la búsqueda de contenidos audiovisuales que complementarían los contenidos recogidos en el temario de la asignatura. Como resultado se obtuvo la ampliación del espacio virtual de la misma, mediante la incorporación del vídeo educativo titulado "Por cuatro esquinitas de nada" ${ }^{17}$ como recurso didáctico.

A continuación, se definieron las tareas que realizarían los estudiantes en el marco de esta actividad, detallándose el plan de trabajo a realizar por ellos. Dicho plan de trabajo incluía el desarrollo de cinco tareas: a) el visionado de los contenidos audiovisuales seleccionados y lectura de los textos temario de la asignatura de un modo comprensivo; b) la puesta en relación de los contenidos audiovisuales y textuales; c) la redacción de una breve presentación de la actividad, con una extensión de entre 200 y 300 palabras, que incluyese un título alternativo a los contenidos audiovisuales objeto de estudio que tuviese relación con el aprendizaje de los adultos; d) la redacción de cinco ensayos breves, con una extensión de entre 200 y 300 palabras, correspondientes a los tópicos del temario; e) la redacción de una conclusión final que

17 Puede encontrarse en el siguiente enlace: $h t t p: / / w w w . y o u t u b e . c o m / w a t c h ? v=D B j k a \_z Q B d Q$ 
incluyese un comentario personal sobre los contenidos audiovisuales utilizados como recurso complementario y su aplicación en la práctica profesional-educativa.

Por último, el diseño de la actividad incluyó la elaboración de un instrumento de recogida de datos orientado a proporcionar información para evaluar la satisfacción de los estudiantes con la actividad y aspectos de gran interés para la docencia, como son: la adquisición, personalización y control del propio aprendizaje a través de la actividad, la motivación suscitada por la propia actividad y las novedades metodológicas introducidas a lo largo del desarrollo de la asignatura. El instrumento diseñado fue un cuestionario semi-estructurado que comprendía 17 preguntas cerradas y siete abiertas. En el diseño del mismo se tuvo en cuenta que su aplicación sería como encuesta electrónica integrada en la plataforma online aLF de la UNED, con objeto de que fuese accesible a todos los estudiantes y permitir la sistematización, la recogida de información y exportación de los datos a formato digital .csv; esto, a su vez, facilitó la depuración de los mismos y la conformación de una base de datos apta para ser utilizada en cualquier software estadístico.

Vínculos fue la última propuesta metodológica de la asignatura "El aprendizaje de las personas adultas en la sociedad del conocimiento" en el curso 2012/13, por lo que su desarrollo por parte de los estudiantes tuvo lugar en el último mes del primer semestre. La actividad fue realizada por el conjunto de la población (diez sujetos) que formaban parte de la primera promoción que participaba en la especialidad de "Calidad de vida en personas adultas y mayores" del Máster Oficial de Intervención Educativa en Contextos Sociales que se imparte en la Facultad de Educación de la UNED. Para su ejecución, el alumnado contó con la plataforma aLF como medio de acceso a los recursos didácticos facilitados por el equipo docente y medio de comunicación con sus miembros integrantes con objeto de obtener apoyo tutorial.

Finalizada la realización de la actividad, por parte de los estudiantes y después de que se publicaran las actas con las calificaciones en la asignatura, se procedió a la evaluación de la misma, mediante el cuestionario diseñado para ello. En esta fase participó un $60 \%$ del alumnado de la asignatura (un total de 6) de modo voluntario previa solicitud de su participación y garantía de anonimato.

\section{Evaluación de la propuesta metodológica}

A continuación, se facilita el análisis de la información aportada por los estudiantes en el cuestionario de valoración de la actividad.

El análisis realizado se basa en la complementariedad de los paradigmas de investigación cuantitativo y cualitativo. En ambos casos, se realiza un análisis de naturaleza descriptiva. Para el análisis descriptivo de los datos cuantitativos (frecuencias y porcentajes), se utilizó la aplicación Excel del paquete informático de Microsoft Office 2011 para Mac. Respecto al análisis descriptivo de los datos cualitativos, se 
codificaron las respuestas abiertas para su posterior aplicación siguiendo el procedimiento de construcción de categorías de Pérez Juste (2006).

\subsection{Datos cuantitativos}

En este apartado se analizan las valoraciones dadas por los estudiantes en las diecisiete preguntas cerradas del cuestionario. Las valoraciones realizadas por los estudiantes siguen una escala tipo Likert de 6 categorías (1 nada, 6 completamente).

La información recabada apunta que la actividad Vínculos ha resultado para los estudiantes una muy buena experiencia, puesto que ha permitido desarrollar su capacidad de elaborar y relacionar información y su sentido crítico, pues el $100 \%$ han estado totalmente de acuerdo con ello (categoría 6). Por otro lado, los estudiantes consideran que el desarrollo de esta actividad ha contribuido a una mayor motivación para el aprendizaje (categorías $5-40 \%$ - y $6-60 \%$-) ; ha generado una mayor implicación en el proceso de aprendizaje (categorías 5 -20\%- y $6-80 \%$-); ha promovido el desarrollo de la capacidad de selección de información (categorías 4 -20\%-. 5 $-40 \%$ - y $6-40 \%$-); ha aumentado la capacidad de elaborar y relacionar información (categoría 6 -100\%-) así como del sentido crítico (categoría 6 -100\%-) (véanse Gráfi$\cos 1,2$ y 3$)$.

Del mismo modo, la actividad ha proporcionado herramientas y destrezas para aprender los contenidos de la asignatura (categorías $5-40 \%$ - y $6-60 \%$-) y ha permitido a los estudiantes relacionar los contenidos teóricos de la asignatura con la práctica educativa de las personas adultas (categorías $4-20 \%-.5-20 \%$ - y $6-60 \%$-) (véanse Gráficos 4 y 5).

En otro orden, el $80 \%$ de los estudiantes valoran en la categoría 6 el video colgado en la plataforma como una buena herramienta para presentar la actividad. El $20 \%$ restante se sitúa en la categoría 5. Idéntica puntuación se obtiene en la valoración de esta forma de trabajo en relación con la evaluación de la asignatura (véanse Gráficos 6 у 7$)$.

La autoevaluación de los estudiantes sobre aspectos relativos a la búsqueda de información, selección de la información, y producto final de la actividad es alta (entre 7.6 y 8 puntos de media) (véase Tabla 1 ).

Las actuaciones del equipo docente han sido valoradas de forma satisfactoria por los estudiantes: forma en que se ha planteado la tarea (categorías $5-20 \%$ - y $6-80 \%$-), $\mathrm{y}$ viabilidad de la tarea teniendo en cuenta el tiempo y el esfuerzo requerido (categorías $5-20 \%$ - y $6-80 \%$-). La relación coste beneficio ha sido valorada de forma más baja (categorías 4 -20\%- y 6 -80\%-) (véanse Gráficos 8, 9 y 10). 
Gráfico 1. La realización de la actividad Vínculos me ha supuesto una mayor motivación hacia el aprendizaje

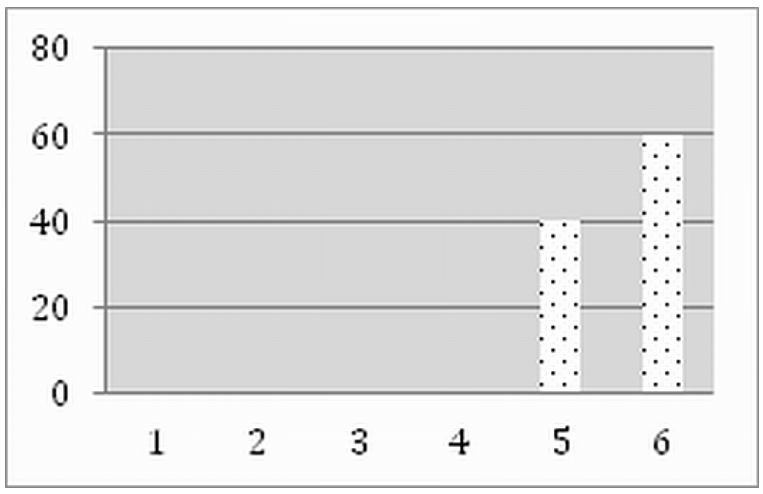

Gráfico 2. La realización de la actividad vínculos me ha permitido mayor implicación el proceso de aprendizaje

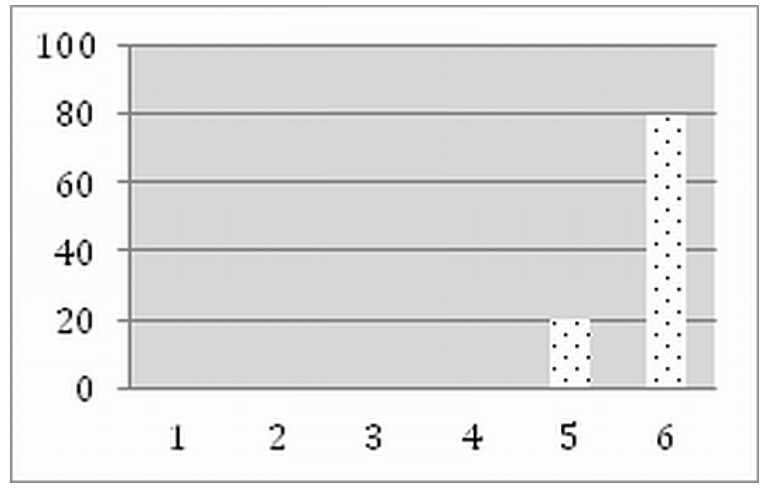

Gráfico 3. La realización de la actividad VINCULOS me ha permitido el desarrollo de la capacidad de selección de información

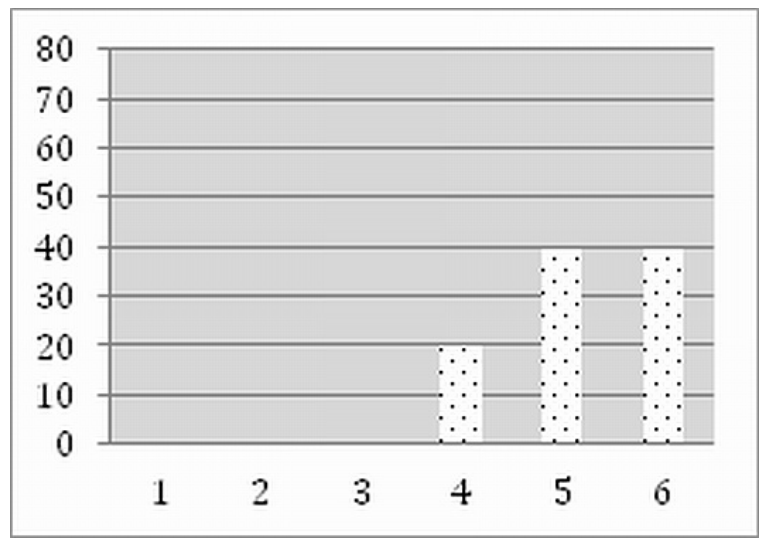


Gráfico 4. La realización de la actividad VÍNCULOS me ha proporcionado herramientas y destrezas necesarias para aprender los contenidos de la asignatura

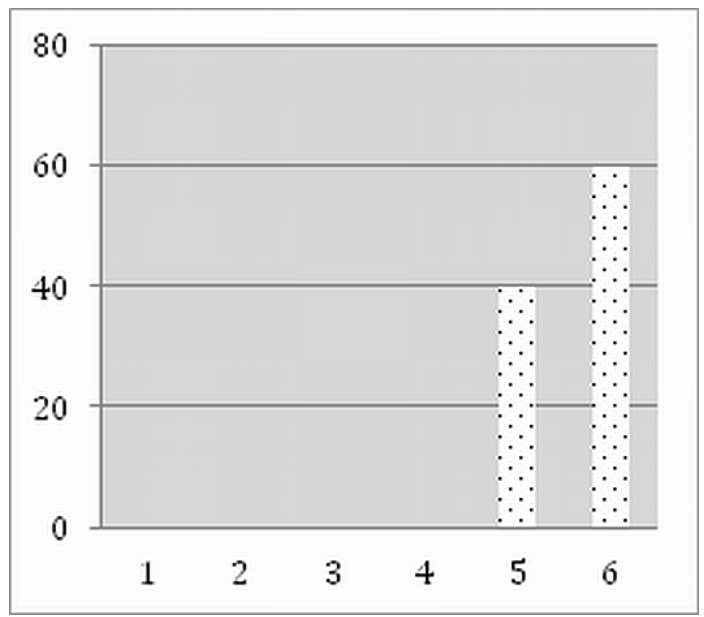

Gráfico 5. La realización de la actividad VÍNCULOS me ha permitido relacionar los contenidos de la asignatura con aspectos de la práctica educativa con personas adultas

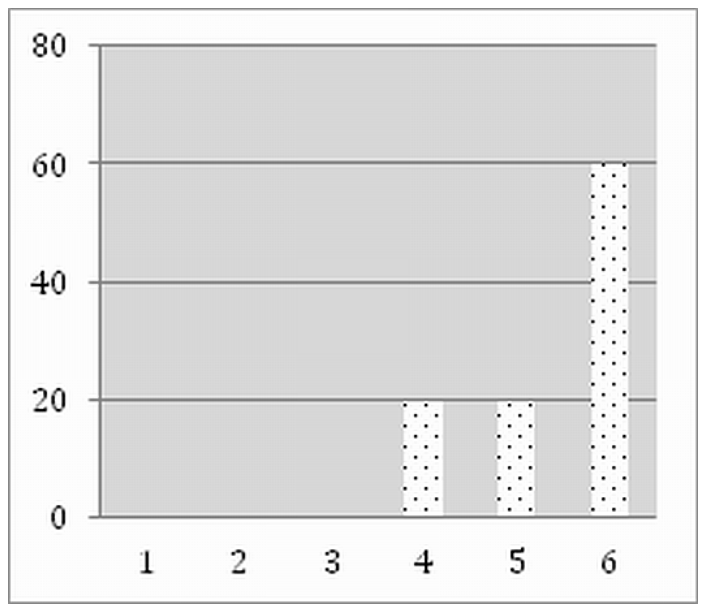


Gráfico 6. ¿En qué medida considera que el video colgado para la realización de la actividad VINCULOS que se encuentra en la plataforma es una buena herramienta para presentar la actividad?

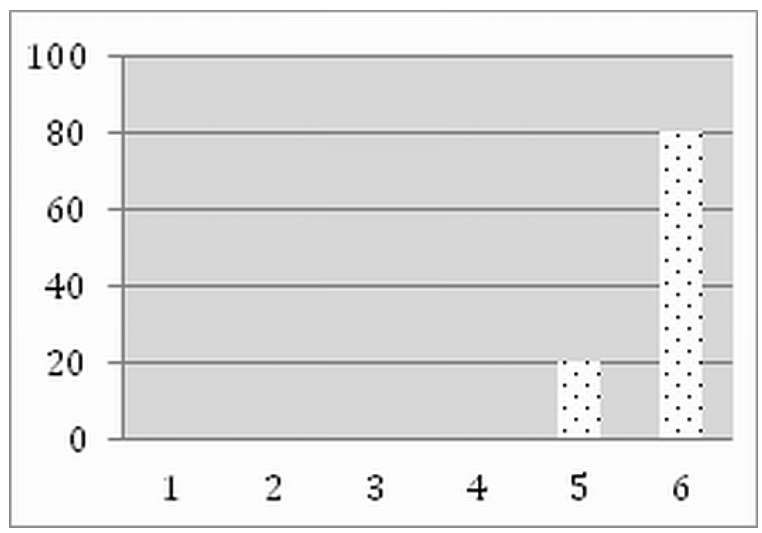

Gráfico 7. ¿Cómo valora esta forma de trabajo en relación con la evaluación de la asignatura?

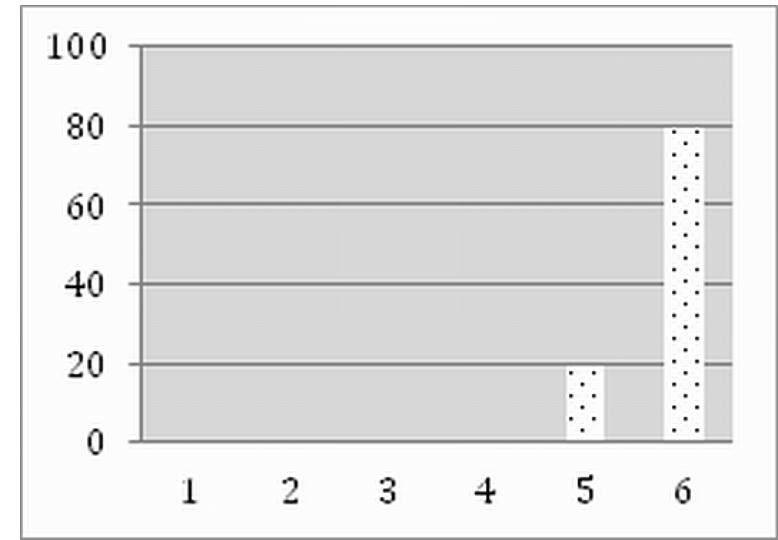


Gráfico 8. Valore a continuación las siguientes actuaciones por parte del Equipo Docente: La forma en que se ha planteado la tarea (claridad de las indicaciones de la Guía, vídeo, etc.).

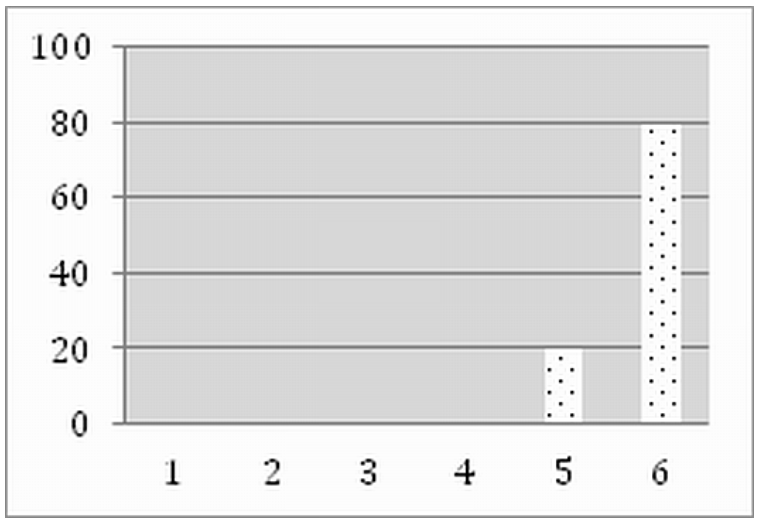

Gráfico 9. Valore a continuación las siguientes actuaciones por parte del Equipo Docente: La viabilidad de la tarea, teniendo en cuenta el tiempo y el esfuerzo requerido

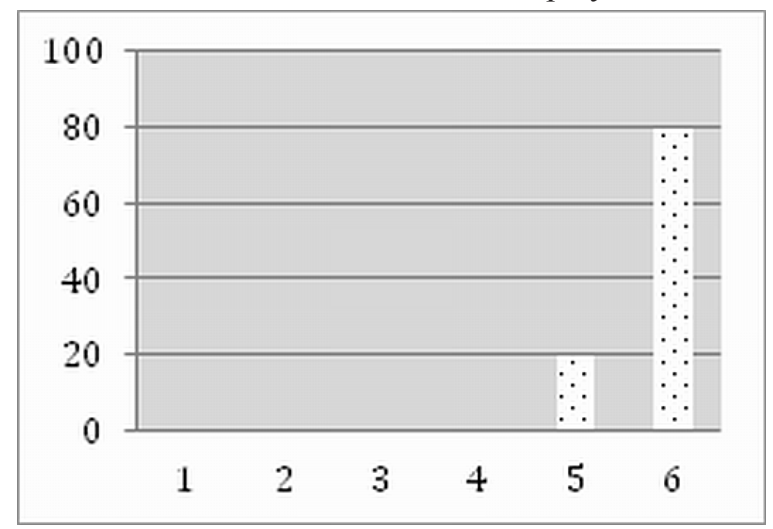

Gráfico 10. Valore a continuación las siguientes actuaciones por parte del Equipo Docente:

La relación coste-beneficio que supone realizar esta tarea

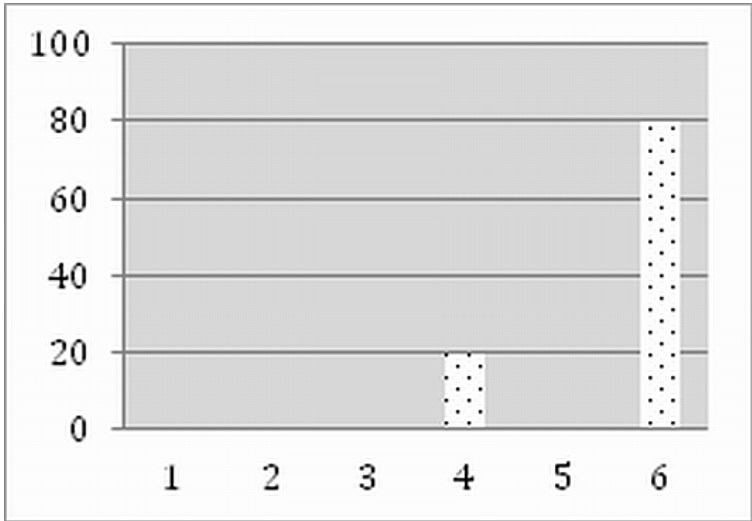


Tabla 1. Autoevaluación

\begin{tabular}{lccccc}
\hline \multicolumn{1}{c}{ Ítems } & N & Mín. & Máx. & Media & Desv. típ. \\
\hline $\begin{array}{l}\text { Realización de una búsqueda de } \\
\text { información acorde a los plantea- } \\
\text { mientos que figuran en la Guía de }\end{array}$ & 5 & 7,00 & 10,00 & 8 &, 8944 \\
\begin{tabular}{l} 
Estudio \\
\hline $\begin{array}{l}\text { Selección de información rele- } \\
\text { vante para mi futuro profesional/ }\end{array}$
\end{tabular} & 5 & 3,00 & 10,00 & 7,6 & 2,497 \\
investigador & 5 & 4,00 & 10,00 & 7.6 & 1.959 \\
\hline $\begin{array}{l}\text { El producto final de la actividad } \\
\text { considero que se ajusta a una } \\
\text { calificación de... }\end{array}$ & & & & & \\
\hline
\end{tabular}

\subsection{Datos cualitativos}

El análisis cualitativo de las respuestas aportadas por los estudiantes a las siete preguntas abiertas del cuestionario arroja las siguientes conclusiones.

En primer lugar, con la realización de la actividad los estudiantes señalaron que esperaban conseguir, comprender y ampliar los contenidos de la materia, objetivos que consideran han conseguido y ampliado con el aprendizaje adicional de la aplicación práctica de la materia y el desarrollo de estrategias meta-cognitivas (véanse Tablas 2, 3 y 4 ).

En segundo lugar, el vídeo 'Por cuatro esquinitas de nada' ha contribuido al establecimiento de procesos de reflexión, pues ha sido definida por los estudiantes como: una buena herramienta para hablar sobre temas sociales y una forma sencilla de comprender groso modo la realidad de la educación de adultos; también al aumento de la motivación, dado que los estudiantes opinan que propicia: mayor predisposición al aprendizaje y muchas ansias de implicación; así como, al fomento de actitudes y valores, teniendo en cuenta que los estudiantes la consideran: una buena herramienta educativa para analizar valores sociales, que permite profundizar mi sensibilidad a la hora de enseñar o de observar el aprendizaje ajeno (véase Tabla 5).

La contribución de la información colgada en la plataforma en el producto final de la actividad ha sido valorada de forma positiva por los estudiantes, ya que les ha permitido la clarificación de los contenidos y la comprensión de la actividad, además del aumento de la motivación y la construcción de aprendizajes.

Por otra parte, la mayoría de los estudiantes no han encontrado dificultades en la realización de la actividad Vínculos y lo han expresado a través de frases como: no he tenido ninguna dificultad; la actividad me ha parecido bastante sencilla y las dificultades estaban "en mi cabeza". Pero en cuanto me senté a hacerla, supe que podría, y, es más, me senti muy feliz haciéndola; no he tenido dificultades, fue cuestión de tener claro lo que en los temas se hablaba. El resto de los estudiantes que sí consideran haber encontrado ciertas dificultades, las ubican fundamentalmente en el 
establecimiento de relaciones entre el contenido teórico y el práctico, y las expresan a través de frases como: en algunas ocasiones, me costó relacionar ciertos contenidos de la materia con los ciertos contenidos del vídeo; al tiempo, que en la realización de un esfuerzo de reflexión y profundización, que describen de la siguiente manera: superar mis propias barreras mentales y tener que salir de mi zona de comodidad y esforzarme por pensar y profundizar en este tema; considero que la actividad exigía una importante capacidad de reflexión y relación de ideas (véase Tabla 6).

En el último apartado del cuestionario 'Añada comentarios u observaciones' todos los estudiantes han mostrado su satisfacción por la actividad, los recursos ofrecidos en la asignatura y la dinámica de trabajo desarrollada por el equipo docente.

Tabla 2. ¿Qué esperaba conseguir con la realización de la actividad Vínculos?

\begin{tabular}{|l|l|}
\hline \multicolumn{1}{|c|}{ Categoría } & \multicolumn{1}{c|}{ Descripciones de los alumnos. } \\
\hline $\begin{array}{l}\text { Comprensión de } \\
\text { los contenidos de la } \\
\text { materia }\end{array}$ & $\begin{array}{l}\text { Analizar en profundidad todos los temas y entender sus contenidos. } \\
\text { Mejorar mi comprensión de los temas. }\end{array}$ \\
\hline $\begin{array}{l}\text { Comprensión de la } \\
\text { aplicación práctica de } \\
\text { la asignatura }\end{array}$ & $\begin{array}{l}\text { Emocionarme con una historia preciosa que no deja de ser la difícil } \\
\text { realidad que muchos viven (hemos vivido) en el día a día, que además } \\
\text { se puede aplicar a muchísimos campos de la realidad, tal como } \\
\text { sucede con la asignatura, donde, dependiendo del lugar en que nos } \\
\text { pongamos, podemos ser perfectamente Cuadraditos o Redonditos. } \\
\text { He aprendido más sobre el aprendizaje en adultos mayores en cuanto } \\
\text { a un contexto social. }\end{array}$ \\
\hline $\begin{array}{l}\text { Desarrollo de } \\
\text { estrategias meta } \\
\text { cognitivas }\end{array}$ & $\begin{array}{l}\text { He conseguido ver que soy capaz de reflexionar, de madurar, de } \\
\text { disfrutar con unos contenidos, incluso de sintetizar y relacionar } \\
\text { conceptos de aquí y de allá, algo que no veía muy claro que era capaz } \\
\text { de hacer. } \\
\text { He descubierto un poco más a mí mismo. } \\
\text { Creo que me sorprendió como podía sacar tantos significados a un } \\
\text { video con tan poca duración. } \\
\text { Agudizar mi habilidad para relacionar ideas. }\end{array}$ \\
\hline \multicolumn{2}{|c}{}
\end{tabular}


Tabla 3. ¿Qué ha conseguido con la actividad VÍNCULOS?

\begin{tabular}{|l|l|}
\hline \multicolumn{1}{|c|}{ Categoría } & \multicolumn{1}{c|}{ Descripciones de los alumnos. } \\
\hline $\begin{array}{l}\text { Comprensión de } \\
\text { los contenidos de la } \\
\text { materia }\end{array}$ & $\begin{array}{l}\text { Analizar en profundidad todos los temas y entender sus contenidos. } \\
\text { Mejorar mi comprensión de los temas. }\end{array}$ \\
\hline $\begin{array}{l}\text { Comprensión de la } \\
\text { aplicación práctica } \\
\text { de la asignatura }\end{array}$ & $\begin{array}{l}\text { Emocionarme con una historia preciosa que no deja de ser la difícil } \\
\text { realidad que muchos viven (hemos vivido) en el día a día, que además } \\
\text { se puede aplicar a muchísimos campos de la realidad, tal como } \\
\text { sucede con la asignatura, donde, dependiendo del lugar en que nos } \\
\text { pongamos, podemos ser perfectamente Cuadraditos o Redonditos. } \\
\text { He aprendido más sobre el aprendizaje en adultos mayores en cuanto } \\
\text { a un contexto social. }\end{array}$ \\
\hline $\begin{array}{l}\text { Desarrollo de } \\
\text { estrategias meta } \\
\text { cognitivas }\end{array}$ & $\begin{array}{l}\text { He conseguido ver que soy capaz de reflexionar, de madurar, de } \\
\text { disfrutar con unos contenidos, incluso de sintetizar y relacionar } \\
\text { conceptos de aquí y de allá, algo que no veía muy claro que era capaz } \\
\text { de hacer. } \\
\text { He descubierto un poco más a mí mismo. } \\
\text { Creo que me sorprendió como podía sacar tantos significados a un } \\
\text { video con tan poca duración. } \\
\text { Agudizar mi habilidad para relacionar ideas. }\end{array}$ \\
\hline
\end{tabular}

Tabla 4. ¿Considera que se han cumplido sus expectativas? Indique por qué.

\begin{tabular}{|l|l|}
\hline \multicolumn{1}{|c|}{ Categoría } & \multicolumn{1}{c|}{ Descripciones de los alumnos. } \\
\hline $\begin{array}{l}\text { Análisis, aplicación } \\
\text { contenidonsión de los }\end{array}$ & $\begin{array}{l}\text { Porque pude analizar los contenidos de la materia y buscarle un sentido } \\
\text { profesional en cuanto a mi futuro laboral. } \\
\text { Me ha dado una amplia perspectiva de los contenidos de la materia. } \\
\text { Mi mayor expectativa era aprender y así fue. }\end{array}$ \\
\hline $\begin{array}{l}\text { Aumento de la } \\
\text { motivación }\end{array}$ & $\begin{array}{l}\text { Ha aumentado mis ansias por saber, por conocer, he buscado en la web } \\
\text { información sobre el cuento, otras opiniones... Me ha encantado. }\end{array}$ \\
\hline $\begin{array}{l}\text { Desarrollo de } \\
\text { estrategias meta } \\
\text { cognitivas }\end{array}$ & $\begin{array}{l}\text { Me ha hecho pararme a pensar mientras preparaba la asignatura y la actividad } \\
\text { en mi realidad y en la de muchas personas. } \\
\text { He conseguido comprender, reflexionar y relacionar ideas que considero } \\
\text { importantes para la comprensión global de los temas. }\end{array}$ \\
\hline
\end{tabular}


Tabla 5. ¿Qué le ha aportado el video "Por cuatro esquinitas de nada..."?

\begin{tabular}{|l|l|}
\hline Categoría & Descripciones de los alumnos. \\
\hline Reflexión & $\begin{array}{l}\text { Buena herramienta para hablar sobre temas sociales. } \\
\text { Tiempo de meditación y reflexión, y el resultado de estas. } \\
\text { Una forma sencilla de comprender groso modo la realidad de la } \\
\text { educación de adultos. }\end{array}$ \\
\hline $\begin{array}{l}\text { Aumento de la } \\
\text { motivación }\end{array}$ & $\begin{array}{l}\text { Mayor predisposición al aprendizaje. } \\
\text { Muchas ansias de implicación. }\end{array}$ \\
\hline Actitudes y valores & $\begin{array}{l}\text { Buena herramienta educativa, ya que en ella se pueden analizar muchos } \\
\text { valores sociales que aparecen en el vídeo. } \\
\text { Profundizar mi sensibilidad a la hora de enseñar o de observar el } \\
\text { aprendizaje ajeno. }\end{array}$ \\
\hline
\end{tabular}

Tabla 6. ¿Qué dificultades ha tenido para realizar la actividad Vínculos?

\begin{tabular}{|l|l|}
\hline Categoría & Descripciones de los alumnos. \\
\hline Sin dificultad & $\begin{array}{l}\text { No he tenido ninguna dificultad. } \\
\text { La actividad me ha parecido bastante sencilla. } \\
\text { Las dificultades estaban "en mi cabeza". Pero en cuanto me senté a hacerla, } \\
\text { supe que podría, y, es más, me sentí muy feliz haciéndola. } \\
\text { No he tenido dificultades, fue cuestión de tener claro lo que en los temas se } \\
\text { hablaba. } \\
\text { No se me ha presentado ninguna dificultad. }\end{array}$ \\
\hline $\begin{array}{l}\text { Relación con } \\
\text { los contenidos }\end{array}$ & $\begin{array}{l}\text { En algunas ocasiones, me costó relacionar ciertos contenidos de la materia } \\
\text { con los ciertos contenidos del vídeo. }\end{array}$ \\
\hline $\begin{array}{l}\text { Reflexión y } \\
\text { profundización }\end{array}$ & $\begin{array}{l}\text { Superar mis propias barreras mentales y tener que salir de mi zona de } \\
\text { comodidad y esforzarme por pensar y profundizar en este tema. } \\
\text { Considero que la actividad exigía una importante capacidad de reflexión y } \\
\text { relación de ideas. }\end{array}$ \\
\hline
\end{tabular}

\section{Conclusiones}

Con objeto de formular conclusiones y propuestas de mejora, se ha considerado adecuado utilizar la técnica DAFO para realizar un análisis holístico de la propuesta metodológica después de su primer curso de aplicación.

El propósito de esta técnica es el de identificar aquellas debilidades, amenazas, fortalezas y oportunidades de la situación objeto de estudio que pueden afectar a la consecución de los objetivos previamente plateados. En el caso de la presente propuesta metodológica, el análisis interno de la misma ha permitido identificar las fortalezas y debilidades de su diseño, desarrollo y evaluación, mientras que el análisis 
externo ha posibilitado la detección de aquellos aspectos vinculados al marco global en el que se ha desarrollado (asignatura, itinerario del Máster y recursos de apoyo utilizados) y que pueden actuar como amenazas y oportunidades. Por tanto, dificultar o posibilitar la consecución de los objetivos.

Los profesores del equipo docente vinculado al desarrollo de la experiencia metodológica han actuado como expertos en la identificación de las debilidades, amenazas, fortalezas y oportunidades de la misma, y lo han hecho a través de una sesión de trabajo en la que se utilizó el brainstorming como técnica de identificación y en la que se tomó como punto de referencia la hipótesis de trabajo y los objetivos de la experiencia. A continuación, facilitamos el resultado de esa sesión de trabajo.

Las debilidades de la propuesta metodológica identificadas han sido: a) únicamente se ha ofertado un vídeo como contenido audiovisual para trabajar los contenidos de la asignatura; b) el papel del alumnado en el desarrollo de la experiencia metodológica ha sido muy reactivo, limitado a trabajar el material audiovisual propuesto a través del planteamiento definido por el equipo docente; c) el reducido tamaño de la muestra de alumnos que participaron en la experiencia y que actuaron como evaluadores de la misma; d) haber ubicado el desarrollo de la evaluación de la experiencia metodológica por parte de los alumnos fuera del cronograma de desarrollo de la asignatura (una vez emitidas las actas de la asignatura); e) la imposibilidad de explotar los datos obtenidos de la evaluación mediante análisis no descriptivos, debido al reducido tamaño de la muestra participante en la experiencia y en la evaluación; y f) la ausencia de algún tipo de refuerzo que estimulase la participación del alumnado en la evaluación de la experiencia.

Con respecto a las fortalezas, se han identificado las siguientes: a) la incorporación de un nuevo tipo de recurso didáctico a la asignatura, el contenido audiovisual; b) situar al alumnado en el centro del proceso de aprendizaje, al buscar estimular su reflexión y pensamiento crítico a través de las actividades planteadas; c) el uso de la plataforma aLF de la UNED, por su potencial para facilitar el acceso a un gran número de recursos didácticos y de hacerlo de una manera universal y equitativa para el conjunto del alumnado; d) haber documentado todo el proceso de diseño, desarrollo y evaluación de la experiencia metodológica, lo que ha permitido tener mucho material para trabajar en las propuestas de mejora; e) el desarrollo de un instrumento de evaluación propio y haber contado con la posibilidad de realizar una aplicación piloto del mismo en el marco del primer curso de la asignatura; y f) la evaluación de la propuesta metodológica se ha apoyado tanto en datos de carácter cuantitativo como de naturaleza cualitativa, lo que ha permitido enriquecer las explicaciones descriptivas con la interpretación que los participantes en la experiencia metodológica realizan de sus aspectos claves.

Los factores externos, vinculados a la asignatura, el itinerario del Máster en los que se contextualiza el desarrollo de la experiencia metodológica y a los recursos de apoyo utilizados, que se han identificado como potenciales amenazas son: a) la ausencia de rigor en la gran cantidad de material audiovisual que se puede localizar 
en Internet como material educativo; b) el tiempo necesario para realizar un buen filtro del material educativo que se puede encontrar en Youtube para trabajar la temática del aprendizaje con personas adultas; c) la reciente creación del Máster que da lugar a que el número de alumnos inscrito en la asignatura sea reducido; d) la ausencia de cultura de evaluación de las propuestas metodológicas desarrolladas en otras asignaturas de estos estudios de postgrado; y e) la baja tasa de respuesta que suelen tener los procesos de encuestación vía on-line y el elevado tiempo de dedicación que es necesario para mejorarla.

Con relación, a las oportunidades identificadas, se destacan: a) el potencial de Internet, concretamente de Youtube, para localizar nuevos materiales audiovisuales de carácter educativo; b) el potencial de los estudiantes como recurso de la asignatura, implicándoles más activamente en la misma mediante la búsqueda y selección de materiales audiovisuales complementarios al propuesto por el equipo docente; c) dado que el Máster se encuentra en sus inicios, es un buen momento para la experimentación de propuestas metodológicas y la aplicación piloto de las mismas; d) los procesos de evaluación general impulsados por la dirección del Máster pueden enmarcar el desarrollo de cultura evaluativa de las propias propuestas metodológicas entre el conjunto del profesorado; y e) el soporte virtual de la experiencia metodológica facilita la exportación del modelo de la actividad a otras asignaturas del Máster.

En definitiva, es posible señalar que, la propuesta Vinculos ha tratado de dar respuesta a los planteamientos del paradigma tecnológico y de la educación a distancia, considerando las nuevas formas de comunicación audiovisual que se sustentan en la Web 2.0 (De-Juanas y Diestro, 2012). Asimismo, esta actividad contempla las posibilidades de un método de enseñanza centrado en el aprendizaje de los estudiantes que se ajusta a los requerimientos del Espacio Europeo de Educación Superior (De Juanas, 2010; Diestro, 2010; González-Olivares, Cruz y Varela, 2011). De tal modo, se ha valorado positivamente el efecto motivador del contenido audiovisual y se ha partido de las ideas previas de los estudiantes. Aspectos que redundan en sus propias creencias epistemológicas (De-Juanas y Beltrán, 2012).

Con todo, se han considerado algunas líneas de actuación futuras que pueden servir para enriquecer el proceso de enseñanza-aprendizaje de la actividad Vínculos. De tal modo, con la finalidad de dar cabida a otros recursos audiovisuales, se estima necesario involucrar al conjunto de estudiantes en la búsqueda de nuevos documentos audiovisuales de contenido educativo que puedan ser utilizados en la formación de personas adultas.

En otro sentido, se contempla la posibilidad de la interacción entre iguales mediante un sistema de aprendizaje compartido y/o cooperativo (Iglesias, Lozano y Martínez, 2013). Para arbitrar esta fórmula, se tienen en cuenta las funcionalidades de la plataforma de enseñanza a distancia aLF. Entre ellas, la más ajustada es la herramienta Foro. Se trata de crear un espacio virtual de debate orientado a la participación y colaboración entre los estudiantes de un modo bidireccional. En este foro, los estu- 
diantes tendrán habilitada la posibilidad de compartir archivos de un gran tamaño, enlaces y textos.

Finalmente, valorando la popularidad de las redes sociales, se plantea un nuevo escenario en el que los estudiantes utilicen estos medios para compartir, divulgar y construir el conocimiento generado en la actividad y en la asignatura. Por ello, se puede implantar un sistema de participación de estudiantes y equipo docente a través de redes sociales que puede ser evaluado pormenorizadamente por el número de mensajes publicados sobre la actividad y por la calidad de su contenido.

\section{Bibliografía}

AREA, M. Y RIBEIRO, M.T. (2012). "De lo sólido a lo líquido: las nuevas alfabetizaciones ante los cambios culturales de la Web 2.0". En: Comunicar 38, 13-20.

CABERO, J. (2004). "No todo es Internet: Los medios audiovisuales e informáticos como recursos didácticos". En: Comunicación y Pedagogía, 20019-23.

DE-JUANAS, A. (2010). "Aprendices y competencias en el Espacio Europeo de Educación Superior”. En: Revista de Psicología y Educación, 5, 171-186.

DE-JUANAS, A. Y BELTRÁN, J.A. (2012). "Creencias epistemológicas de los estudiantes de Pedagogía y Ciencias de la Educación”. En: Revista de Psicodidáctica, 17(1), 179-198.

DE-JUANAS, A. Y DIESTRO, A. (2012). "Empleo de los medios sociales en educación superior: una nueva competencia docente en ciernes". En: REDU. Revista de Docencia Universitaria, 10 (2), 365-379.

DELORS, J. (1996). La educación encierra un tesoro. Informe a la UNESCO de la Comisión Internacional sobre la Educación para el Siglo XXI. Ediciones UNESCO.

DIESTRO, A. (2010). "El horizonte educativo en Europa". En: Foro de Educación, 12, 3-6.

GONZÁLEZ-OLIVARES, CRUZ, R. Y VARELA, V. (2011). "Proyecto Aprende: práctica formativa innovadora". En: Revista de Psicología y Educación, 6,79-91.

IGLESIAS, M.J., LOZANO, I., MARTÍNEZ, M. A. (2013). "La utilización de plataformas virtuales para el desarrollo de metodologías participativas: análisis de una experiencia en Educación Superior". En: REDU. Revista de Docencia Universitaria, 11 (2), 333-352.

LOZANO, A. Y COVELO, J.M. (2009). "Educando en la sociedad del conocimiento. El empleo de las TIC en el aula (retos y posibilidades)". En Hekademos, $n^{\circ} 2$, 55-70.

MASTERMAN, L. (1993): La enseñanza de los medios de comunicación. Ediciones de la Torre. Madrid.

SEVILLANO GARCÍA, Ma L. (2009). Competencias para el uso de herramientas virtuales en la vida, trabajo y formación permanentes. Pearson. Madrid. 


\section{Los autores}

Ana Eva Rodríguez Bravo es profesora del Departamento Teoría de la Educación y Pedagogía Social de la Universidad Nacional de Educación a Distancia. Doctora en Ciencias de la Educación por la Universidad Complutense de Madrid. En la actualidad es investigadora del Grupo de Intervención Socioeducativa (UNED) y del Grupo de Investigación sobre Políticas Educativas Supranacionales de la (UAM).

Beatriz Tasende Mañá es colaboradora de la Cátedra Unesco de Educación a Distancia. Licenciada en Ciencias de la Educación por la Universidad Complutense de Madrid y en Psicología por la Universidad Nacional de Educación a Distancia. Entre sus temas de investigación destacan las tecnologías en la educación.

Ángel Luis González Olivares es profesor del Departamento de Teoría de la Educación y Pedagogía Social de la Universidad Nacional de Educación a Distancia. Doctor en Ciencias de la Educación por la Universidad Complutense de Madrid. En la actualidad es investigador del Grupo de Intervención Socioeducativa (UNED).

Álvaro Muelas Plaza es profesor del Centro Universitario Villanueva (adscrito a la Universidad Complutense de Madrid). Doctor en Ciencias de la Educación de la Universidad Complutense de Madrid. Psicopedagogo y orientador escolar en el Bachillerato Fomento-Fundación. Entre sus temas de investigación, destacan las estrategias de aprendizaje.

Ángel De-Juanas Oliva es profesor del Departamento de Teoría de la Educación y Pedagogía Social de la Universidad Nacional de Educación a Distancia. Doctor en Ciencias de la Educación por la Universidad Complutense de Madrid. En la actualidad es investigador del Grupo de Competencias profesionales docentes para desarrollar competencias básicas en los alumnos de primaria y secundaria (UCM) y del Grupo de Intervención Socioeducativa (UNED). 